\title{
ORIGINAL
}

\section{MORTALIDAD POR SUICIDIO EN ANDALUCÍA: DISTRIBUCIÓN GEOGRÁFICA Y RELACIÓN CON EL USO DE ANTIDEPRESIVOS, LA ALTITUD Y DESIGUALDADES SOCIOECONÓMICAS}

\author{
José Alameda-Palacios (1), Miguel Ruiz-Ramos (2) y Beatriz García-Robredo (3).
}

(1) Unidad de Gestión Clínica de Salud Mental. Hospital Universitario Virgen Macarena. Servicio Andaluz de Salud. Sevilla. España.

(2) Servicio de Información y Evaluación. Consejería de Igualdad. Salud y Políticas Sociales de la Junta de Andalucía. Sevilla. España.

(3) Servicio de Promoción del Uso Racional del Medicamento. Dirección General de Asistencia Sanitaria y Resultados en Salud. Servicio Andaluz de Salud. Sevilla, España.

\section{RESUMEN}

Fundamentos: Andalucía tiene una mortalidad por suicidio superior a la media española y una evolución temporal discordante con el conjunto del país. El objetivo fue analizar la evolución reciente de la mortalidad por suicidio en Andalucía, su distribución geográfica y la relación con el uso de antidepresivos, la altitud y las desigualdades socioeconómicas.

Métodos: Estudio ecológico cuya unidad de análisis fueron las Zonas Básicas de Salud de Andalucía. Se calcularon las tasas de suicidio ajustadas por edad del periodo 2007-2011 y de 2012. Para analizar las variables asociadas a las diferencias geográficas se midió la correlación mediante el coeficiente de Spearman y se estimaron modelos lineales generalizados.

Resultados: Andalucía tuvo una tasa de mortalidad por suicidio de $7,58 / 100.000$ habitantes/año entre 2007 y 2011, con un intervalo de 0,96 a 27,71. Hubo correlación positiva entre altitud y tasas de suicidio en 2012 $(\mathrm{r}=0,29 ; \mathrm{p}<0,001)$ y en el periodo 2007-2011 $(\mathrm{r}=0,47 ; \mathrm{p}<0,001)$. En $2012 \mathrm{el}$ uso de antidepresivos no se correlacionó con las tasas de suicidio $(\mathrm{r}=0,03$; $\mathrm{p}=0,67)$ pero sí durante los cinco años anteriores $(\mathrm{r}=0,18 ; \mathrm{p}=0,008)$. El Índice de Privación Material tuvo una correlación positiva con las tasas en 2012 $(\mathrm{r}=0,29 ; \mathrm{p}<0,001)$ y de 2007-2011 ( $\mathrm{r}=0,33 ; \mathrm{p}<0,001)$.

Conclusiones: Existe un claro patrón geográfico en la distribución de la mortalidad por suicidio en Andalucía que permanece estable en el período estudiado. La zona central y las de mayor altitud concentran las tasas de suicidio más altas y se asocian con utilización de antidepresivos y mayor privación material.

Palabras clave: Suicidio. Altitud. Antidepresivos. Desigualdades en salud. Factores socioeconómicos. Depresión. Salud mental.

\footnotetext{
Correspondencia

José Alameda Palacios

Unidad de Gestión Clínica de Salud Mental

Hospital Universitario Virgen Macarena

Avenida Dr. Fedriani $\mathrm{n}^{\circ} 3$

41071. Sevilla

jose.alameda.sspa@juntadeandalucia.es
}

\section{ABSTRACT \\ Suicide Mortality in Andalusia, Spain: Geographical Distribution and Relationship with Antidepressants, Altitude and Socioeconomic Inequalities}

Background: The suicide mortality rate in Andalusia is higher than the Spanish average, and it shows a different evolution over time. This study analyzes recent changes of suicide mortality in Andalusia, its geographical distribution and its relation with antidepressant use, altitude and socioeconomic inequality.

Methods: An ecological study of the Basic Health Zones in Andalusia has been conducted. Age-standarized suicide rates in 2012 and during 20072011 were calculated. The correlation among variables and their association with the geographical differences was assessed with Spearman's coefficient and generalized linear models were also estimated.

Results: Andalusia registered 7,58 suicides per 100.000 inhabitants per year from 2007 to 2011 , with a range of 0,96 to 27,71 in the different areas. Positive correlation has been observed between altitude and suicide rates in $2012(\mathrm{r}=0,29 ; \mathrm{p}<0,001)$ and during the period 2007-2011 $(\mathrm{r}=0,47 ; \mathrm{p}<0,001)$. Antidepressant use in 2012 was not correlated with suicide rates in that year $(\mathrm{r}=0,03 ; \mathrm{p}=0,67)$, but a positive correlation was observed with the precedent five years suicide rates $(\mathrm{r}=0,18 ; \mathrm{p}=0,008)$. The Deprivation Index had a positive correlation with suicide rates in $2012(\mathrm{r}=0,29 ; \mathrm{p}<0,001)$ and in 2007-2011 (r=0,33; $\mathrm{p}<0,001)$

Conclusions: There is a clear geographical pattern in the distribution of suicide mortality in Andalusia and it remains stable over time. Central areas and in those with the highest altitude concentrate the highest suicide rates, which are associated with a larger use of antidepressants and also with higher levels of material deprivation.

Keywords: Suicide. Altitude. Antidepressant agents. Socioeconomic factors. Health inequalities. Depression. Mental health.

DOI: 


\section{INTRODUCCIÓN}

El suicidio es un importante problema social y de salud pública que está recibiendo una atención creciente en los últimos años. En 2012 se registraron más de 800.000 muertes por esta causa en todo el mundo, lo que representa una mortalidad de 11,4 por 100.000 habitantes ${ }^{1}$.

El suicidio presenta una gran variabilidad geográfica y temporal que está siendo analizada en numerosos estudios ecológicos en los que se examina la relación entre las diferencias en las tasas de suicidio y diversos factores socioeconómicos ${ }^{2-6} \mathrm{o}$ medioambientales $^{7-10}$. Estos estudios se están añadiendo recientemente a los que hasta hace poco centraban su atención en la relación existente entre las variaciones en la mortalidad por suicidio $y$ el uso de antidepresivos ${ }^{11-13}$.

En España se ha informado de tendencias temporales decrecientes desde los años 90 hasta finales de la década de los años 2000. Hay variaciones geográficas estables en el tiempo entre las diferentes zonas del país, que muestran un claro patrón de tasas más elevadas en zonas económicamente deprimidas, entre las que se encuentra Andalucía ${ }^{14,15}$, que no ha presentado la tendencia descendente observada en el conjunto de España ${ }^{16}$. Recientemente, el Instituto Nacional de Estadística (INE) ha comunicado que en 2012, el número de suicidios aumentó en España un $11,3 \%$ con respecto al año anterior, alcanzando una tasa de 7,6 por cada 100.000 habitantes, la más alta desde $2005^{17}$.

Las diferencias geográficas de las tasas de mortalidad por suicidio en Andalucía han mostrado también patrones estables en el tiempo en diversos niveles geográficos y grupos de edad y sexo. Se conocen las tasas específicas por grupos de edad y sexo a nivel municipal hasta $2012^{18}$, y las tasas ajustadas por edad a nivel de distritos sanitarios para hombres y mujeres hasta $2007^{19}$. La relación de las variaciones geográficas con sus posibles determinantes no ha sido estudiada hasta ahora y no se dispone de un dato unitario que refleje la carga global de suicidio en Andalucía y en sus diferentes áreas geográficas.

El objetivo del estudio fue analizar la evolución reciente de la mortalidad por suicidio en Andalucía, su distribución geográfica y la relación con el uso de antidepresivos, la altitud sobre el nivel del mar y las desigualdades socioeconómicas.

\section{MATERIAL Y MÉTODO}

Se llevó a cabo un estudio ecológico descriptivo de la mortalidad por suicidio en el ámbito geográfico de la Comunidad Autónoma de Andalucía, cuya unidad de análisis fueron las 216 zonas básicas de salud (ZBS) en las que se divide según el Mapa de Atención Primaria de Salud de Andalucía ${ }^{20}$. Se examinó la mortalidad registrada en el año 2012 y se comparó con el promedio del periodo 2007-2011.

Para ello se obtuvieron las muertes por suicidio de cada municipio de Andalucía por edad y sexo registradas por el Instituto de Estadística y Cartografía de Andalucía (IECA) en los años 2007 a 2012, incluyendo como causas de muerte las comprendidas entre los códigos X60 a X84 de la 10 revisión de la Clasificación Internacional de Enfermedades. Los datos municipales se agregaron por ZBS. Igualmente, las cifras de población para cada zona básica se agregaron a partir de los datos municipales del Censo de Población y Viviendas de 2011 publicado por el INE ${ }^{21}$. Se calcularon las tasas ajustadas por edad, tomando como referencia la población estándar europea, para el conjunto de Andalucía y para cada ZBS en el año 2012 y en el periodo 2007-2011 en hombres, mujeres y el conjunto de la población. Las diferencias encontradas se representaron mediante mapas de coropletas con los cuartiles de las variables. La información cartográfica para la elaboración de los mapas se obtuvo del sistema de Datos Espaciales de Referencia de Andalucía del IECA $^{22}$. 
Los datos de prescripción de antidepresivos corresponden a los registrados en la base de datos de facturación de recetas oficiales del sistema sanitario público andaluz del año 2012. El consumo se expresó en Dosis Diarias Definidas (DDD) de los medicamentos contenidos en el grupo N06A de la clasificación Anatomical Therapeutic Chemical (ATC). Las tasas de consumo de antidepresivos se expresaron como DDD por 1.000 habitantes y día (DHD). La identificación del sexo y la asignación a una ZBS sólo fue posible para el $91,33 \%$ de las prescripciones de 2012, aunque el cálculo de la DHD del conjunto de Andalucía sí se realizó computando el $100 \%$ de las recetas facturadas.

La altitud sobre el nivel del mar de los municipios se obtuvo del Sistema de Información Multiterritorial de Andalucía del IECA $^{23}$. Para estimar la altitud de cada ZBS se calculó la media de los municipios que la constituyen.

Las diferencias socioeconómicas se estimaron mediante el Índice de Privación Material (IPM), que combina información sobre analfabetismo, desempleo y porcentaje de trabajadores manuales de los municipios de España ${ }^{24}$. Para las 55 ZBS que tenían un solo municipio se utilizó el propio valor del índice, mientras que para las compuestas por varias localidades, se obtuvo una aproximación al mismo calculando la media del IPM de los municipios que las constituyen.
La asociación entre las tasas de suicidio y los factores estudiados se midió mediante el coeficiente de correlación de Spearman. Para el análisis multivariante, dado que los datos no tenían una distribución normal y que la regresión de Poisson presentaba sobredispersión, se optó por ajustar modelos lineales generalizados de regresión quasipoisson que permitieran estimar el grado de dispersión. Se utilizó el programa $\mathrm{R}$ versión 3.1.1 para el análisis estadístico y los mapas se realizaron con el programa Geoclip.

\section{RESULTADOS}

Entre 2007 y 2012 se registraron en Andalucía 4.412 suicidios, de los que 3.466 (78,6\%) fueron en hombres y 946 en mujeres (21,4\%). En 2012 la tasa ajustada de suicidio para el conjunto de la población fue de 8,19 por 100.000 habitantes/año (IC95\%: 7,60-8,78) y en el periodo 20072011 de 7,58 100.000 habitantes/año (IC 95\%: 7,33-7,83). La tasa de 2012 fue por tanto un $8 \%$ más alta que el promedio de los cinco años anteriores, aunque la diferencia no fue estadísticamente significativa.

La tasa ajustada para toda Andalucía en hombres fue de 13,37 (IC 95\%: 12,2914,45 ) en 2012 y de 12,28 (IC 95\%: 11,8312,73 ) en el periodo 2007-2011. En mujeres la tasa ajustada fue de 3,37 (IC 95\%: 2,84-3,89) en 2012 y de 3,23 (IC 95\%: 2,99-3,46) en los cinco años anteriores.

Tabla 1

Tasas ajustadas de suicidio de las ZBS de Andalucía. Suicidios / 100000 habitantes / año

\begin{tabular}{|l|r|r|r|r|r|c|}
\hline & Mínimo & Percentil 25 & Mediana & Percentil 75 & Máximo & $\begin{array}{c}\text { Coeficiente } \\
\text { de variación }\end{array}$ \\
\hline Tasa de suicidio 2007-2011 & 0,96 & 5,89 & 7,94 & 11,13 & 27,21 & 0,51 \\
\hline Tasa de suicidio 2007-2011 hombres & 1,24 & 9,65 & 13,81 & 18,40 & 39,93 & 0,50 \\
\hline Tasa de suicidio 2007-2011 mujeres & 0 & 0,85 & 2,79 & 4,62 & 19,51 & 1,01 \\
\hline Tasa de suicidio 2012 & 0 & 3,29 & 7,8 & 12,94 & 55,93 & 0,98 \\
\hline Tasa de suicidio 2012 hombres & 0 & 4,58 & 12,42 & 21,36 & 110,48 & 1,06 \\
\hline Tasa de suicidio 2012 mujeres & 0 & 0 & 0 & 4,74 & 40,23 & 1,94 \\
\hline
\end{tabular}




\section{Figura 1}

Distribución geográfica de la tasa ajustada de suicidio para toda la población en el periodo 2007-2011

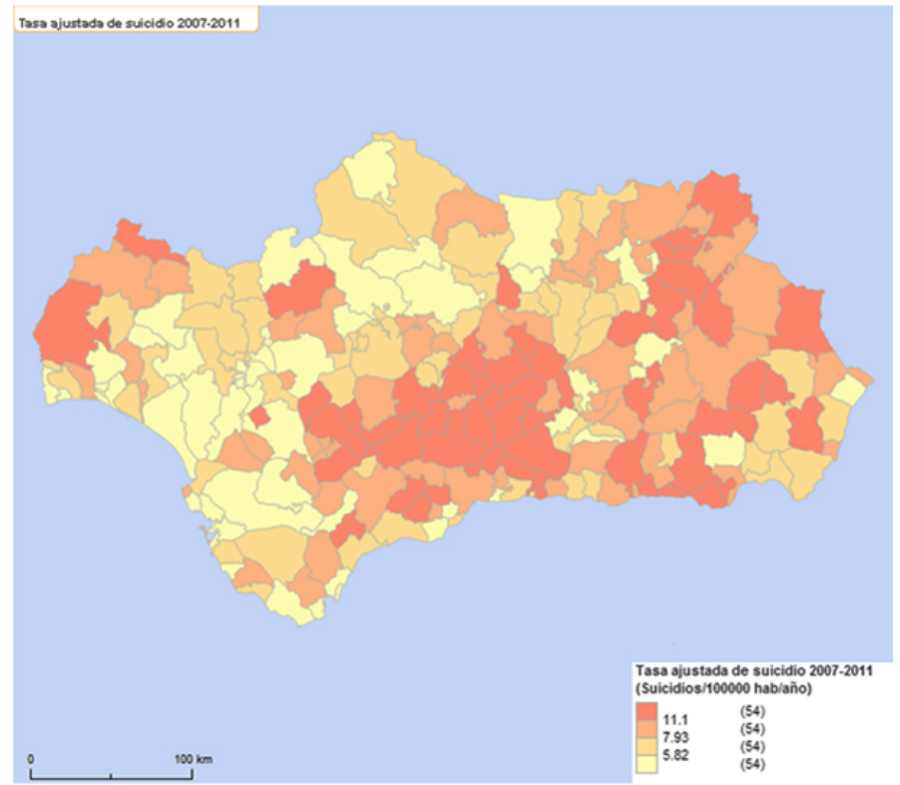

Figura 2

Distribución geográfica de la tasa ajustada de suicidio para toda la población en 2012

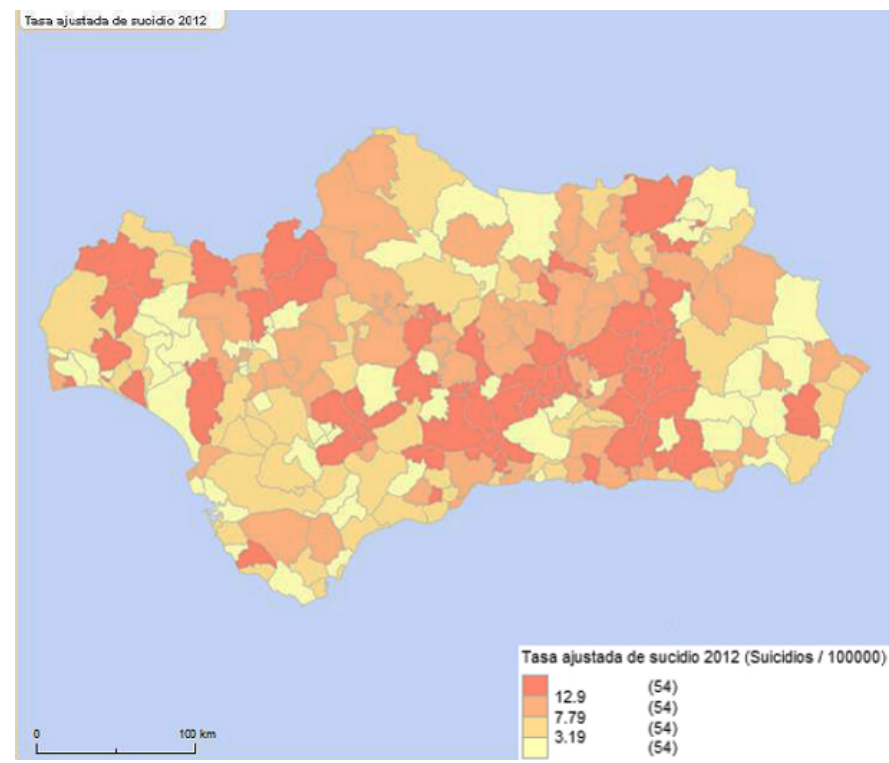


Las tasas ajustadas de las 216 ZBS de Andalucía en ambos periodos se resumen en la tabla 1. La variabilidad de las tasas de mujeres fue prácticamente el doble que la de los hombres y el conjunto de la población, tanto en 2012 como en el periodo 2007-2011. Las tasas de 2012 presentaron igualmente el doble de variabilidad que las estimadas para el periodo 2007-2011. El rango intercuartílico de las tasas del conjunto de la población fue de 9,65 en 2012 y de 5,23 en el periodo 2007 2011.

Las variaciones geográficas de las tasas ajustadas registradas en las ZBS se presentan en las figuras 1 y 2 mediante mapas en los que el primer cuartil tiene el color más claro y el cuarto el más oscuro. En el periodo 2007-2011, 50 ZBS presentaron una tasa ajustada al menos un 50\% superior a la de Andalucía, y en 27 la tasa fue más del doble. En 2012, fueron 59 las ZBS con una tasa de más de 1,5 veces la andaluza y 33 las que al menos la doblaban.
Las ZBS con tasas más altas se encontraron mayoritariamente en la zona central de Andalucía y en las áreas más montañosas de todas las provincias. Mientras que las ZBS con tasas más bajas se situaron predominantemente en la depresión bética y en el litoral. En la figura 1 se aprecia que la mayoría de las ZBS con tasas de suicidio en el periodo 2007-2011 incluidas en el cuarto cuartil se agrupaban en un área compacta situada en la confluencia de las provincias de Sevilla, Córdoba, Jaén, Granada y Málaga. Fuera de esta región central, el resto de ZBS con tasas altas se distribuía casi sin excepción por las zonas de mayor altitud sobre el nivel del mar de todas las provincias. En 2012 (figura 2), la distribución era muy parecida, aunque las tasas presentaron mayor variabilidad y la zona central es menos compacta, apareciendo más ZBS con tasas elevadas dispersas en las provincias de Sevilla, Huelva, Granada y Almería.

La figura 3 muestra la razón de tasas entre la mortalidad por suicidio en 2012 y el perio-

Figura 3

Razón de tasas ajustadas de suicidio 2012/2007-11

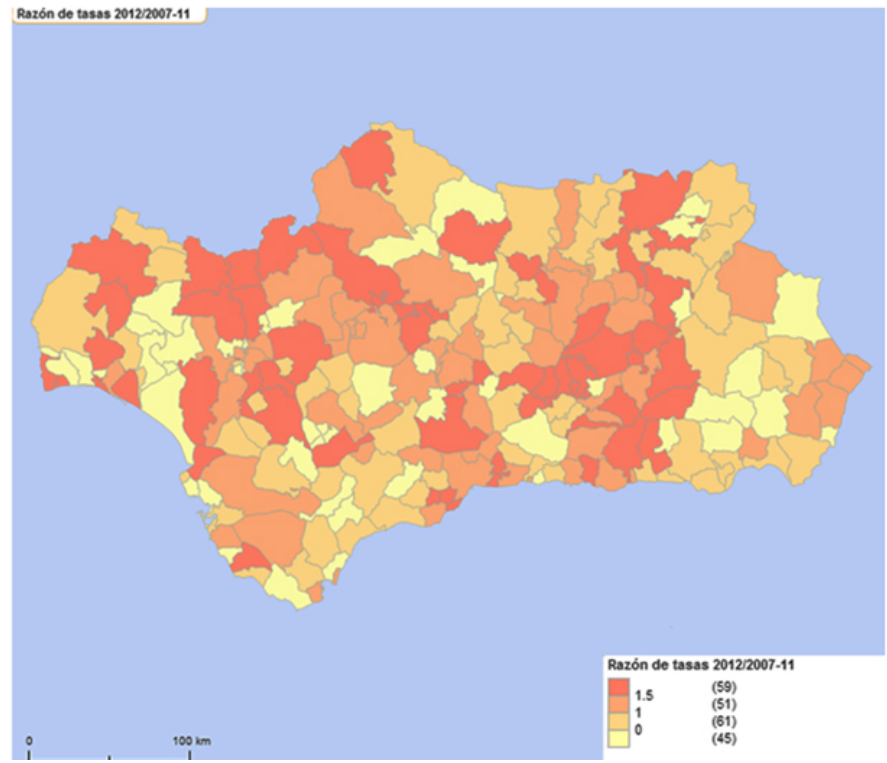




\begin{tabular}{|c|c|c|c|c|c|}
\hline \multicolumn{6}{|c|}{$\begin{array}{c}\text { Tabla } 2 \\
\text { Características de las zonas básicas de salud de Andalucía }\end{array}$} \\
\hline & Mínimo & Percentil 25 & Mediana & Percentil 75 & Máximo \\
\hline Población & 3.576 & 13.057 & 21.716 & 33.853 & 698.042 \\
\hline Hombres & 1.841 & 6.801 & 10.896 & 17.132 & 332.203 \\
\hline Mujeres & 1.735 & 6.438 & 10.823 & 17.004 & 365.839 \\
\hline DHD Antidepresivos & 23,07 & 41,57 & 51,16 & 61,69 & 90,25 \\
\hline DHD Hombres & 11,98 & 20,42 & 25,45 & 30,66 & 53,92 \\
\hline DHD Mujeres & 33,97 & 62,90 & 77,93 & 93,48 & 134,45 \\
\hline Índice Privación Material & $-0,27$ & 0,93 & 1,56 & 2,10 & 3,49 \\
\hline Altitud media & 2 & 98 & 352 & 629 & 1.327 \\
\hline
\end{tabular}

Figura 4

Distribución geográfica de la DHD de antidepresivos por en Andalucía en 2012

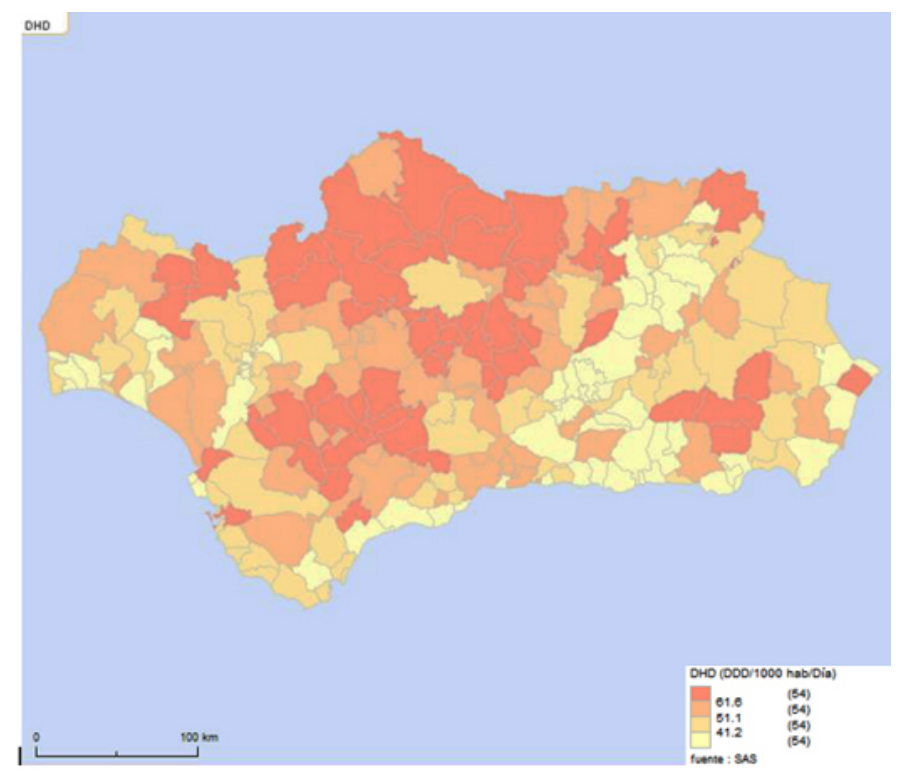

do 2007-2011. Los límites de las coropletas no se corresponden en este caso con los de los cuartiles, sino que se han fijado en $0,1 \mathrm{y}$ 1,5. De este modo, el color blanco representa las 45 ZBS en las que no hubo suicidios en 2012 y, por tanto, tienen una razón de tasas de 0 . El color amarillo muestra las 61 ZBS con razón de tasas inferior a $1 \mathrm{y}$, por tanto, con menos suicidio en 2012 que en el promedio de los cinco años anteriores. En color anaranjado aparecen las 51 ZBS con razón de tasas entre 1 y 1,5 que presentaron hasta un $50 \%$ más suicidio en 2012. Y, por último, en rojo se muestran las 59 ZBS en las que en 2012 la mortalidad por suicidio fue al menos 1,5 veces la de 2007-2011.

Las características de las ZBS se resumen en la tabla 2, en la que se puede observar que son muy desiguales en lo que se refiere al tamaño de la población, oscilando entre 3.576 y 69.8042 habitantes. La distribución geográfica de las tasas de consumo de antidepresivos en 2012 se presenta en la figura 4, en la que 


\begin{tabular}{|l|c|c|}
\hline \multicolumn{3}{|c|}{ Tabla 3 } \\
Correlación entre las tasas de suicidio y los principales factores estudiados
\end{tabular}

\begin{tabular}{|l|c|c|c|c|}
\hline \multicolumn{5}{|c|}{ Tabla 4 } \\
& Coeficiente $(\beta)$ & $\begin{array}{c}\text { Error } \\
\text { estándar }\end{array}$ & $p$ & $\begin{array}{c}\text { Parámetro } \\
\text { de dispersion }(\Phi)\end{array}$ \\
\hline Conjunto de la población 07-11 & & & & 1,71 \\
\hline Altitud (x 100 m) & 0,62 & 0,09 & & \\
\hline DHD Antidepresivos (x 10) & 0,48 & 0,22 & 0,033 & \\
\hline IPM & 0,70 & 0,37 & 0,063 & \\
\hline Hombres 07-11 & & & & 2,65 \\
\hline Altitud (x 100 m) & 1,01 & 0,14 & & \\
\hline DHD Antidepresivos (x 10) & 1,16 & 0,58 & 0,047 & \\
\hline IPM & 1,72 & 0,55 & 0,002 & \\
\hline Mujeres 07-11 & & & & 2,95 \\
\hline Altitud (x 100 m) & 0,19 & 0,07 & 0,009 & \\
\hline DHD Antidepresivos (x 10) & 0,18 & 0,12 & 0,156 & \\
\hline IPM & -0.03 & 0,31 & 0,935 & \\
\hline Conjunto de la población 2012 & & & & \\
\hline Altitud (x 100 m) & 0,76 & 0,19 & & \\
\hline DHD Antidepresivos (x 10) & $-0,45$ & 0,45 & 0,315 & \\
\hline IPM & 2,62 & 0,74 & & \\
\hline
\end{tabular}

se aprecia un patrón geográfico en el que la mayor utilización de antidepresivos se da predominantemente en la provincia de Córdoba y la más baja en las de Jaén y Granada. La DHD de antidepresivos del conjunto de Andalucía en 2012 fue de 55,08 DDD por 1.000 habitantes/día.

En la tabla 3 se muestra la correlación entre los factores estudiados y las tasas ajustadas de suicidio de 2012 y del periodo 2007-2011. La altitud sobre el nivel del mar de las ZBS presentó la correlación positiva más fuerte, sobre todo con la tasa estimada para el periodo 2007-2011. El IPM presentó igualmente correlación positiva con ambas tasas. La DHD de antidepresivos en 2012 tuvo una correla- ción positiva débil con la tasa de suicidio en 2007-2011 pero no con la de 2012. El tamaño de la población de la ZBS tuvo una correlación negativa con la tasa de suicidio del periodo 2007-2011 ( $\mathrm{r}=-0,33, \mathrm{p}<0,001)$, pero no hubo correlación estadísticamente significativa con las tasas de $2012(\mathrm{r}=-0,07$, $\mathrm{p}=0,29)$.

El análisis multivariante mostró que las tasas de suicidio en el periodo 2007-2011 fueron mayores a mayor altitud, mayor privación material y más consumo de antidepresivos (tabla 4). En el modelo ajustado para el conjunto de la población, que explicó el 26,93\% de la variabilidad (calculada como proporción de la deviance restada del modelo nulo), y 
que tuvo un moderado grado de sobredispersión $(\phi=1,71)$, el coeficiente estimado para la altitud suponía que para una diferencia de altitud de 500 metros (aproximadamente el rango intercuartílico de esta variable) la tasa de suicidio aumentaba en 3,08 por 100.000 habitantes/año (IC 95\%: 2, 19-3,99). Un cambio de 10 puntos en la DHD de antidepresivos (aproximadamente la mitad del rango intercuartílico) suponía un aumento en la tasa de suicidio de 0,48 (IC 95\%: 0,030,93 ) y un cambio de 1 punto en el IPM (casi el $90 \%$ del rango intercuartílico) producía un cambio en la tasa de 0,70 (IC 95\%: -0,051,45). El modelo ajustado para los hombres explicaba el 29,1 de la variabilidad, aunque con mayor sobredispersión $(\phi=2,65)$ y estimó un aumento de la tasa de suicidio de 5,04 por 100.000 (IC 95\%: 3,63-6,48) para un incremento de 500 metros de altitud; de 1,16 (IC 95\%: 0,01-2,33) por cada 10 puntos de aumento de la DHD en hombres (que en este caso equivale al rango intercuartílico) y 1,72 (IC 95\%: 0,60- 2,83) para un aumento de 1 punto del IPM. El modelo ajustado para las tasas de suicidio en mujeres explicó un $4,67 \%$ de la variabilidad y la asociación no fue estadísticamente significativa ni en la DHD de antidepresivos ni en el IPM. El modelo de ajuste de tasas en 2012 para el conjunto de la población explicó el 15,9\% de la variabilidad, con un parámetro de dispersión de 6,76 y encontró una asociación estadísticamente significativa con la altitud y el IPM. Para los hombres fue muy similar, aunque con el doble de dispersión y para las mujeres ninguna de las variables resultó estadísticamente significativa, por lo que ninguno de los dos puede considerarse válido y por ello no se muestran en la tabla.

\section{DISCUSIÓN}

La mortalidad por suicidio presenta una gran variabilidad geográfica en Andalucía, con zonas claramente diferenciadas de alta y baja incidencia que se distribuyen con arreglo a un patrón geográfico estable a lo largo del tiempo.
La tasa ajustada de suicidio de Andalucía en 2012 es un $60 \%$ más alta que la registrada ese año en España, que fue de 5,1 casos por 100.000 habitantes $^{1}$. Esto supone que más de la mitad de las ZBS tienen una tasa al menos un $50 \%$ por encima de la media española que llega a duplicarse o triplicarse en las áreas con tasas más altas. Por tanto, la magnitud de las diferencias encontradas es considerable y apunta a un exceso de mortalidad por esta causa que es endémico desde hace muchos años en algunas zonas de Andalucía ${ }^{18-19}$. Estos niveles de suicidio en Andalucía en 2012 no suponen diferencias estadísticamente significativas respecto a los cinco años anteriores, como se ha señalado que sí ocurre con el conjunto de España ${ }^{17}$.

Por lo que se refiere a las diferencias geográficas, las tasas de suicidio más altas se asocian con una mayor altitud sobre el nivel del mar, mayor privación material, menor tamaño de la población de la ZBS y un consumo más frecuente de antidepresivos. La altitud, que es el factor más claramente asociado a las variaciones de la mortalidad por suicidio, se asocia también en Andalucía con una mayor ruralidad y con un menor tamaño de las poblaciones de la ZBS, por lo que su relación con el suicidio puede ser un reflejo de otras variables no incluidas en el estudio, tanto socioeconómicas como medioambientales.

Entre las limitaciones de este estudio están las derivadas de las fuentes de información utilizadas. La propia mortalidad por suicidio se sabe que puede estar subregistrada por razones culturales que incluyen el estigma asociado. La tasa estimada para el periodo 2007-2011 es un promedio y no recoge las fluctuaciones que experimentó la mortalidad por suicidio en este periodo, en el que tuvo ascensos y descensos ${ }^{16}$.

El consumo de antidepresivos podría estar sobreestimado, puesto que en este estudio se valoran recetas dispensadas sin poder conocer la adherencia o el cumplimiento terapéutico. Aunque por otra parte, para el cálculo de las DHD de las ZBS solo se pudo analizar 
el $91,33 \%$ de las recetas y solo del sistema sanitario público, ya que se excluyeron mutualidades y medicina privada, por lo que las tasas reales de utilización de antidepresivos son probablemente superiores a las calculadas en este estudio.

El IPM no está disponible para las ZBS y solo se ha podido obtener una aproximación al mismo en las zonas compuestas por más de un municipio. Además, corresponde al año 2001 y no recoge por tanto los posibles efectos de la crisis económica sobre las desigualdades socioeconómicas.

La unidad de análisis elegida tiene la ventaja de poder examinar la relación entre suicidio y uso de antidepresivos con la máxima discriminación posible, pero debido a la heterogeneidad de las ZBS supone también una limitación, ya que la naturaleza de los datos no permitió ajustar modelos válidos para todas las tasas calculadas y los que se ajustaron no recogen bien la extravariabilidad de las ZBS más pequeñas, sobre todo en las tasas de 2012, ya que en el promedio del periodo 2007-2011 esta variabilidad se suaviza en parte. Por ello, con esta unidad de análisis, las estimaciones relativas a un solo año tienen un mayor componente aleatorio y no reflejan bien el patrón geográfico subyacente que se aprecia mejor en las tasas estimadas para el periodo 2007-2011.

Por otra parte, sólo una pequeña parte de las diferencias encontradas quedan explicadas por las variables estudiadas, por lo que es necesario seguir investigando posibles factores relacionados con la variabilidad del suicidio, sobre todo los que puedan ser tan estables en el tiempo como son las diferencias en la mortalidad.

Por lo que se refiere a la relación de los resultados encontrados con hallazgos de estudios anteriores, hay que decir en primer lugar que los trabajos existentes sobre el tema en nuestro medio han encontrado un patrón geográfico muy similar ${ }^{18,19}$, por lo que se puede afirmar que la distribución actual de la morta- lidad por suicidio se mantiene estable al menos desde los años 70 del siglo pasado.

La asociación entre aumento del uso de antidepresivos y reducción del suicidio es controvertida $^{12}$. Si bien es cierto que a finales del siglo pasado y comienzos de este se encontró esa asociación en muchos países ${ }^{11}$, no ocurre lo mismo en otros lugares en los que también aumentó el uso de antidepresi$\operatorname{vos}^{13}$. Recientemente se ha examinado en qué medida mayores niveles de uso de antidepresivos podían jugar un papel protector frente al aumento del suicidio atribuido a factores socioeconómicos, sin encontrar ningún efecto estadísticamente significativo ${ }^{4}$. Los resultados del presente estudio muestran que no hay correlación entre las tasas de suicidio de 2012 y el consumo de antidepresivos en ese mismo año, pero sí una correlación positiva, aunque débil, entre la tasa de suicidio de los años anteriores y el uso de antidepresivos en 2012. Por tanto, es plausible pensar que altos niveles de suicidio inducen mayor consumo de antidepresivos, pero que no se traduce en una reducción del suicidio. Por otra parte, la tasa de uso de antidepresivos encontrada en este estudio para el conjunto de Andalucía en 2012 (55,08 DHD) es significativamente más baja que la de España, que se situaba en 71,79 DHD en $2012^{25}$.

Las desigualdades socioeconómicas medidas a través de distintas variables se han relacionado con las diferencias en las tasas de suicidio entre países o zonas geográficas ${ }^{3-6}, y$ han sido propuestas como explicación de las mayores tasas de Andalucía con respecto a otras zonas de España ${ }^{14,15}$. También en Andalucía se encuentra que al menos una parte de la variabilidad de la mortalidad por suicidio puede asociarse a las desigualdades socioeconómicas medidas a través del IPM. Variables como la tasa de desempleo, la renta per cápita o los niveles de protección social, entre otras muchas que se han relacionado con la mortalidad por suicidio, no han podido ser analizadas en este estudio por no estar disponibles a nivel de las ZBS. 
La correlación positiva entre altitud y suicidio es conocida y se ha constatado en diversos entornos geográficos ${ }^{7,10}$. Aunque se ha señalado la posible influencia de la hipoxia como mecanismo directo a través del cual una mayor altitud aumentaría por sí misma el riesgo de suicidio ${ }^{7,26,27}$, también se ha informado de una interacción entre altitud y concentraciones de litio en el agua potable en relación con la mortalidad por suicidio ${ }^{10}$. En zonas con mayor altitud, el agua presenta menores concentraciones de litio y este factor por sí mismo tiene una correlación negativa con las tasas de suicidio ${ }^{8,9}$. En el caso de Andalucía, la correlación que se ha encontrado entre suicidio y altitud es clara, resultando el factor más relacionado con la variabilidad del suicidio.

En conclusión, existe un claro patrón geográfico en la distribución de la mortalidad por suicidio en Andalucía que permanece estable en el tiempo. La zona central y las de mayor altitud concentran las tasas de suicidio más altas, asociándose con más consumo de antidepresivos y mayor privación material. En estas zonas se dan tasas de suicidio muy superiores a la media española de forma constante en las últimas décadas.

\section{BIBLIOGRAFÍA}

1. World Health Organization. Preventing suicide: A global imperative. Geneva: WHO; 2014.

2. Chang S-S, Stuckler D, Yip P, Gunnell D. Impact of 2008 global economic crisis on suicide: time trend study in 54 countries. BMJ. 2013;347:f5239.

3. Reeves A, McKee M, Stuckler D. Economic suicides in the Great Recession in Europe and North America. Br J Psychiatry. 2014; 205:246-7

4. Reeves A, McKee M, Gunnell D, Chang S-S, Basu $\mathrm{S}$, Barr B, et al. Economic shocks, resilience, and male suicides in the Great Recession: cross-national analysis of 20 EU countries. Eur J Public Health. 2014 Oct 6. En prensa, disponible en: http://dx.doi.org/10.1093/eurpub/ cku168

5. Laanani M, Ghosn W, Jougla E, Rey G. Impact of unemployment variations on suicide mortality in Western European countries (2000-2010). J Epidemiol Community Health. 2015; 69:103-9.
6. Phillips JA, Nugent CN. Suicide and the Great Recession of 2007-2009: The role of economic factors in the 50 U.S. states. Soc Sci Med. 2014;116:22-31

7. Kim N, Mickelson JB, Brenner BE, Haws CA, Yurgelun-Todd DA, Renshaw PF. Altitude, Gun Ownership, Rural Areas, and Suicide. Am J Psychiatry. 2011;168:4954.

8. Kapusta ND, Mossaheb N, Etzersdorfer E et al. Lithium in drinking water and suicide mortality. Br J Psychiatry. 2011;198:346-350.

9. Blüml V, Regier MD, Hlavin G et al. Lithium in the public water supply and suicide mortality in Texas. J Psychiatr Res. 2013;47:407-11.

10. Helbich M, Blüml V, Leitner M, Kapusta ND. Does altitude moderate the impact of lithium on suicide? A spatial analysis of Austria. Geospat Health. 2013;7:20918.

11. Ludwig J, Marcotte DE, Norberg K. Anti-depressants and suicide. J Health Econ. 2009; 28:659-676.

12. Isacsson G, Rich CL, Jureidini J, et al. The increased use of antidepressants has contributed to the worldwide reduction in suicide rates. Br J Psychiatry. 2010;196:429433.

13. Zahl P-H, De Leo D, Ekeberg Ø, et al. The relationship between sales of SSRI, TCA and suicide rates in the Nordic countries. BMC Psychiatry. 2010;10:62.

14. Álvaro-Meca A, Kneib T, Gil-Prieto R, Gil de Miguel A. Epidemiology of suicide in Spain, 1981-2008: A spatiotemporal analysis. Public Health. 2013;127:380-5.

15. Salmerón D, Cirera L, Ballesta M, Navarro-Mateu F. Time trends and geographical variations in mortality due to suicide and causes of undetermined intent in Spain, 1991-2008. J Public Health (Oxf). 2013;35:237-45.

16. Alameda-Palacios J, Ruiz-Ramos M, García-Robredo B. Suicidio, prescripción de antidepresivos y desempleo en Andalucía. Gac Sanit. 2014; 28: 309-2.

17. Instituto Nacional de Estadística. Defunciones según la Causa de Muerte. Año 2012. Disponible en: http:// www.ine.es/prensa/np830.pdf

18. Ocaña-Riola R, Mayoral-Cortés JM, Sánchez-Cantalejo C, et al. Atlas Interactivo de Mortalidad en Andalucía (AIMA). Rev. Esp. Salud Publica. 2008;82:379-394.

19. Ruiz Ramos M. Tendencias de la mortalidad por suicidio en Andalucía. En: Recomendaciones sobre la Detección, Prevención e Intervención de la Conducta Suicida. Granada: EASP Consejería de Salud; 2010. p 73-84. 
20. Servicio Andaluz de Salud. Mapa de Atención Primaria de Salud de Andalucía. Sevilla: Consejería de Salud; 2003.

21. Instituto Nacional de Estadística. INEbase/Demografía y población/Cifras de población y censos demográficos/Censos de Población y Viviendas 2011. Disponible en: http://www.ine.es/censos2011_datos/ cen11_datos_inicio.htm

22. Instituto de Estadística y Cartografía de Andalucía. Datos espaciales de referencia de Andalucía.Disponible en: http://www.juntadeandalucia.es/institutodeestadisticaycartografia/DERA/

23. Instituto de Estadística y Cartografía de Andalucía. Sistema de Información Multiterritorial de Andalucía (SIMA). Disponible en: http://www.juntadeandalucia. es/institutodeestadisticaycartografia/sima/index2.htm

24. Sánchez-Cantalejo C, Ocaña-Riola R, Fernández A. Deprivation index for small areas in Spain. Soc Indic Res. 2008; 89: 259-273.

25. Agencia Española de Medicamentos y Productos Sanitarios. Enero 2015. Utilización de medicamentos antidepresivos en España durante el periodo 20002013. Disponible en: http://www.aemps.gob.es/medicamentos UsoHumano/observatorio/docs/antidepresivos-2000-2013.pdf

26. Young SN. Elevated incidence of suicide in people living at altitude, smokers and patients with chronic obstructive pulmonary disease and asthma: possible role of hypoxia causing decreased serotonin synthesis. J Psychiatry Neurosci. 2013; 38: 423-6.

27. Huber RS, Coon H, Kim N, Renshaw PF, Kondo DG. Altitude is a risk factor for completed suicide in bipolar disorder. Med Hypotheses. 2014; 82: 377-81. 